\title{
Evaluación para aprender: Las ideas de los profesores de ciencias sociales sobre la evaluación del entendimiento causal
}

\author{
J. Alonso-Tapia ${ }^{1}$, F. Asensio ${ }^{2}$, I. López ${ }^{3}$, \\ N. Carriedo ${ }^{4}$, F. Rycheck ${ }^{5}$
}

\footnotetext{
${ }^{1}$ Facultad de Psicología, Universidad Autónoma de Madrid ${ }^{2}$ Inst. de Educación Secundaria María Zambrano, Leganés (Madrid) ${ }^{3}$ Inst. de Educación Secundaria Joaquín Araujo, Fuenlabrada (Madrid)

${ }^{4}$ Departamento de Psicología Evolutiva y de la Educación, Universidad Nacional de Educación a Distancia, Madrid ${ }^{5}$ Director General, Facultad Católica de Rondonia, Brasil
}

España / Brasil

Jesús Alonso Tapia. Universidad Autónoma de Madrid, Campus de Canto Blanco. 28049 Madrid, Spain. E-mail: jesus.alonso@uam.es

(C) Education \& Psychology I+D+i and Editorial EOS (Spain) 


\section{Resumen}

El principal objetivo de este trabajo, llevado a cabo como parte de una serie dirigida a la identificación de las ideas de los profesores sobre la evaluación, fue identificar las ideas de los profesores de ciencias sociales acerca de la manera adecuada de evaluar el entendimiento causal cuando se evalúa para aprender. Tras establecer el modelo psicológico del cual las ideas de los profesores deben ser evaluadas, se les pidió a 68 profesores de enseñanza secundaria que diseñaran situaciones de evaluación adecuadas para dicho propósito. Los resultados mostraron una convergencia de datos provenientes de Brasil y España e iluminaron características específicas de las ideas de los profesores acerca de la evaluación del entendimiento casual. Por lo tanto son útiles en el diseño de programas de formación de profesores para la evaluación del alumnado.

Palabras clave: evaluación del entendimiento causal; evaluación en las ciencias sociales; evaluación de los conocimientos; pensamiento de los profesores.

Recepción: 07-06-07 Aceptación provisional: 08-10-07 Aceptación definitiva: 23-11-07 